\title{
Comprehensive Analysis of an Unsteady Radiated MHD Natural Convective Nanofluid in the Existence of Magnetic Field Fixed to the Vertical Plate or to the Fluid
}

\author{
Suresh Babu Ramakrishna ${ }^{1 *}$ (D), Sravan Kumar Thavada ${ }^{2}$ (D), Neeraja Gullapalli ${ }^{1}$ (D) \\ 1 Department of Mathematics, M S Ramaiah Institute of Technology, Bangalore-560 054, Karnataka, India; \\ sureshbabu_r80@yahoo.co.in (S.B.R.); gneerajamaths@gmail.com (N.G).; \\ 2 Department of Mathematics, REVA University, Bangalore-560 064, Karnataka, India; thavadasravankumar@gmail.com. \\ (S.K.T.); \\ * Correspondence: sureshbabu_r80@yahoo.co.in;
}

Scopus Author ID 57199323358

Received: 19.12.2020; Revised: 24.01.2021; Accepted: 26.01.2021; Published: 30.01.2021

Abstract: Analytical analysis has been done for the unsteady, natural convective flow of an impulsive water-based nanofluid consisting of nanoparticles $\mathrm{Cu}, \mathrm{Al}_{2} \mathrm{O}_{3}$, and $\mathrm{TiO}_{2}$ through a vertical plate under a uniform magnetic field when the magnetic field fixed to the plate $(K=$ 1 ) or to the fluid $(K=0)$. For this model, we considered a radiative heat flux as well as pressure gradient defined by the Boussinesq's approximation in the energy equation for a nonscattering medium. The Governing PDE's are derived and transformed to dimensionless form and then obtained a closed-form solution using Laplace transform procedure without any limitation. The fluid properties have been discussed for a moving and the static plate through graphs for different water-based nanoparticles and also dimensionless parameters such as volume fraction parameter $(\varnothing)$, radiation $\operatorname{parameter}(N r)$, and magnetic parameter $\left(M^{2}\right)$, Grashof number(Gr) etc. of the physical model.

Keywords: Nanoparticles; $\mathrm{Cu} ; \mathrm{Al}_{2} \mathrm{O}_{3} ; \mathrm{TiO}_{2} ; \mathrm{MHD}$; thermal radiation; volume fraction; vertical plate; Laplace transform technique.

\section{Nomenclature:}

\begin{tabular}{l|l|l|l}
$T$ & Temperature; & $u$ & Velocity along $x$-axis; \\
\hline$\mu_{n f}$ & Dynamic viscosity; & $g$ & Acceleration; \\
\hline$\beta_{n f}$ & $\begin{array}{l}\text { Thermal expansion } \\
\text { coefficient; }\end{array}$ & $q_{r}$ & Radiative heat flux; \\
\hline$\rho_{n f}$ & Density; & $B_{0}$ & Magnetic field; \\
\hline$\sigma_{n f}$ & Electrical conductivity; & $M$ & Magnetic parameter; \\
\hline$k_{n f}$ & Thermal conductivity; & $\mathrm{Pr}$ & Prandtl number; \\
\hline$\left(\rho c_{p}\right)_{n f}$ & Heat capacitance; & $\mathrm{Nr}$ & Radiation parameter; \\
\hline$\phi$ & $\begin{array}{l}\text { Solid volume fraction of } \\
\text { the } \\
\text { nanoparticle; }\end{array}$ & $\mathrm{Gr}$ & Grashof number; \\
\hline$\rho_{s}$ & $\begin{array}{l}\text { Density of the } \\
\text { nanoparticle; }\end{array}$ & $\mathrm{Nu}$ & Nusselt number; \\
\hline$\sigma_{s}$ & $\begin{array}{l}\text { Electrical conductivity of } \\
\text { the } \\
\text { nanoparticle; }\end{array}$ & $\tau$ & Time;
\end{tabular}




\begin{tabular}{l|l|l|l}
\hline$\left(\rho c_{p}\right)_{s}$ & $\begin{array}{l}\text { Heat capacitance of the } \\
\text { nanoparticle; }\end{array}$ & $a_{0}^{\prime}$ & Accelerating parameter; \\
\hline$k_{s}$ & $\begin{array}{l}\text { Thermal conductivity of } \\
\text { the } \\
\text { nanoparticle; }\end{array}$ & $\sigma^{*}$ & $\begin{array}{l}\text { Stefan-Boltzmann } \\
\text { constant; }\end{array}$ \\
\hline$\rho_{f}$ & Density; & $k^{*}$ & $\begin{array}{l}\text { Rosseland absorption } \\
\text { coefficient; }\end{array}$ \\
\hline$\sigma_{f}$ & Electrical conductivity; & Subscripts \\
\hline$\mu_{f}$ & Viscosity; & $n f$ & Nanofluid; \\
\hline$k_{f}$ & Thermal conductivity; & $f$ & Base fluid; \\
\hline $\begin{array}{l}\left(\rho c_{p}\right)_{f} \\
\text { Commons Attribution (CC BY) license (https://creativecommons.org/licenses/by/4.0/). }\end{array}$ & Heat capacitance; & $s$ & Nanoparticles; \\
\hline
\end{tabular}

\section{Introduction}

In recent times, nanotechnology has been increasingly shaping scientists and researchers' important role in manufacturing sciences and engineering. Especially in the pharmaceutical and medical sectors, cancer patient's treatments consist of various radiation and medicines in cooling and heating like reducing heat from CPU's, managing temperatures in nuclear reactors, cars, and thermal flows. In modern times, nanofluids have intrigued researchers and scientists with these main features with many industrial applications. Nanofluids immersed in normal fluids tend to improve their thermal efficiency. Nanofluids are a new type of heat transfer fluids based on nanotechnology caused by dispersing nanometersized elements from 1-100 $\mathrm{nm}$ in standard heat transfer fluids. Nanoparticles have emerged as acceptable candidates for fluid suspension due to their vast external space, lower particle energy, and extraordinary mobility. Nanoparticles (a mixture of Metals, carbon nanotubes, and carbides) have important industrial uses, such as self-propelled sunscreens are more resistant to radiation, car bumpers for weights, rubber bones for more strength, clothing is more stain repellent, and balls are more stable for many sports. Widely, in the era of nanotechnology, where an item is decreasing in size and its characteristics are improving, nano-catalysts have actual usefulness in numerous processes such as compound solid rocket propellants, water refinement, production of biodiesel, drug transfer, and carbon nanotubes production.

In cooling and related technologies, nanofluid is used to solve normal problems with typical slurries, such as sedimentation, pressure drop, clogging, corrosion, and microchannel applicability. This idea was first proposed by Choi [1] and found that owing to the additional thermal conductivity of combined nanoparticles. A nanofluid has much greater thermal conductivity compared to normal fluids. Eastman et al. [2] examined that nanofluid consisting of copper particles in nanosize spread in Ethylene glycol has greater thermal conductivity than pure ethylene glycol or ethylene glycol with a fraction of the same volume. Das et al. [3] studied thermal diffusivity calculation for nanofluids consists of nanoparticles $\mathrm{Al}_{2} \mathrm{O}_{3}$ or $\mathrm{CuO}$ with water as the base fluid using a temperature oscillation technique. Das and Choi [4] discussed convection, boiling, pool boiling of nanofluids, and nanofluid applications and observed that improvement of thermal conductivity is higher for nanofluid compared to ordinary micrometer-sized suspensions. Kuznetsov and Nield [5] examined using Buongiorno's model when the nanofluid particle fraction is passively rather than actively regulated at the border. Srinivas et al. [6] analyzed the impact of wavy channel corrugation on improving heat transfer of an Au-water nanofluid. Chamkha et al. [7] focused on the boundary- 
layer flow of a nanofluid along a plate consisting of a pure fluid under the influence of the magnetic field, heat generation, and suction effects and concluded that the pure fluid had strong effects on thermophysical properties. Jahan et al. [8] examined stability and regression analyses in a practical approach past a permeable stretching sheet over the heated surface.

An electrically conducting fluid flow with an external constraint of the magnetic field can be regulated. The transfer rate can also be controlled. Various industrial applications can be seen in many sciences and technology, viz nuclear cooling reactors, boundary layer control in aerodynamics, plasma studies, petroleum industries, crystal growth, etc. Hence, the authors are received new attention for studying the most general contexts of magnetohydrodynamics (MHD) with the influence of the magnetic fields' external force on electrically conduction fluid. At present, due to the existence of favorable characteristics concerning energy processes, material engineering, and medical operations, magneto-nanofluids have become very significant. Many developments occur at very high temperatures, and comprehensive knowledge of heat transfer requires equipment planning. The process group includes satellites, space aircraft, missiles, generators, and nuclear power plants. This reality draws many researchers' attention to finding such acceptable mixtures with an extreme heat transfer rate. In this work, nanofluid consists of nanoparticle Copper and Titanium oxide and water as base fluid to find dissimilarity in heat improvement and transfer rates for different ranges of base fluid and nanoparticles. In the presence of a revolving frame of reference, Hamad et al. [9] studied nanoparticles' effect (metallic) on the unstable MHD convective flow over an oscillating semi-infinite vertical permeable layer analytically. Simulation of nanofluid and rate of heat transmission among the parallel plates in two phases for major impacts of Brownian motion is being investigated by Sheikholeslami et al. [10]. Impact of heat source on an unstable MHD free convection flow of a Casson fluid past a vertical oscillating plate using finite element analysis studied by Shankar Goud et al. [11] and for a micropolar fluid by Islam et al. [12]. Sheikholeslami et al. [13,14] studied the effects of MHD, Lorentz forces, heat transfer numerically on $\mathrm{A} 12 \mathrm{O} 3$ water, and $\mathrm{CuO}$-water nanofluid flow a semi-annulus enclosure using LBM. In clean fluid and $\mathrm{Cu}$-water nanofluid, Molli et al. [15] studied unsteady electrically conducting heat and mass transfer magneto-hydrodynamic flow with $\mathrm{Cu}$-nanoparticles over a plate with various boundary conditions. Animasaun et al. [16] conducted a comparative study of alumina-water nanofluid flows between $36 \mathrm{~nm}$ and $47 \mathrm{~nm}$ in the presence of the Hall effect.

By considering water as a typical base fluid comprising three dissimilar nanoparticles such as $\mathrm{Cu}, \mathrm{Al}_{2} \mathrm{O}_{3}$, and $\mathrm{TiO}_{2}$, Hussanan et al. [17] analyzed the unstable MHD effect, heat transfer rate past an accelerating plate and found that $\mathrm{Cu}$-water is better than that of $\mathrm{Al}_{2} \mathrm{O}_{3}$ water and $\mathrm{TiO}_{2}$-water nanofluids. Abbas et al. [18] researched heat transfer thermophysical properties for unstable nanofluids consisting of nanoparticles $\mathrm{Cu}, \mathrm{Al}_{2} \mathrm{O}_{3}$, and $\mathrm{TiO}_{2}$ with different shapes, and water as a base fluid past a spinning plate under the action of ohmic, viscous dissipation, Brownian, and thermophoresis diffusion. Hayat et al. [19] examined the attendance of applied magnetic fields. Viscous dissipations of mixed convective peristaltic nanofluid transport consist of Copper, Silver, Gold, and iron oxide. They also stated that the Maxwell is better than the Hamilton model. For three different nanofluids (carbon Nanotubes$\mathrm{Ga}$, Copper-Ga, and Diamond-Ga) in which the amount of Grashof ranges 104 to 106 and the volume fraction from 0.01 to 0.15 , Zhou et al. [20] investigated heat transfer improvement and entropy production of nano (metal) fluid in a heated cavity. Heat transfer development for a time-dependent MHD Oldroyd-B nanofluid consists of SWCNTs. Multi-wall carbon nanotubes (MWCNTs) are immersed in a porous material through an infinite vertical plate studied by 
Anwar et al. [21]. A large number of researchers have given considerable interest to the study of MHD in the presence of radiation owing to a large number of astrophysics and geophysics applications, radio propagation, etc. In the inclined magnetic field's attendance, thermal radiation, Sandeep et al. [22] focused on hydromagnetic unsteady electrically conducting fluid past a moving plate by ramped temperature. Nandkeolyar et al. [23] illustrated MHD flow through radiative heat transfer of an isothermal plate to examine the behavior of ramping at the wall and inclination of the applied magnetic field. He concluded that the free-stream state is more rapid than the flow past. Kumar et al. [24] studied thermal diffusion and radiation effects liquid chemically reacting on an accelerated plate with adjustable temperature under the impact of an invariant magnetic field in two different states. Mishra et al. [25] investigate the effect of radiation and non-uniform heat source on the unstable, magneto-hydrodynamic viscous fluid through a heated plate with time-dependent suction and viscous dissipation.

Chiranjeevi et al. [26] investigated the issue of the MHD boundary layer flow analysis in the presence of thermal absorption with heat generation and chemical reaction past a plate. To simulate the 2D unstable magneto-hydrodynamic flow of an electrically conducting fluid over a permeable plate, a mathematical model has been developed by Suneetha et al. [27] to simulate the value of thermal radiation. Empirical analysis has been done on a nanofluid's thermal transportation characteristics with radiation effect was performed in Turkyilmazoglu et al. [28] and invented that the lowest heat transfer occurs for $\mathrm{TiO}_{2}$ and the highest for $\mathrm{Cu}$. MHD heat transfer flow between two parallel plates was inspected by Sheikholeslami et al. [29] under thermal radiation for both Brownian motion and thermophoresis. Satya Narayana et al. [30] discussed thermal radiation characteristics on the MHD nanofluid flow bounded by a time-oscillating constant frequency with a constant heat source. Using the Laplace transformation technique, Pandit et al. [31] studied chemical reactions and radiation effects on unstable MHD electrically conducting viscous fluid with heat-absorbing over a flat plate. Variable thermophysical properties are studied numerically under the act of radiation and a magnetic field for an unstable Couette copper-water nanofluid by Wakif et al. [32] in the case of two parallel plates by keeping one of them in an accelerated motion. Heikholeslami et al. [33] addressed Lorentz force and radiation's impact on nanoparticles $\left(\mathrm{Al}_{2} \mathrm{O}_{3}-\mathrm{H}_{2} \mathrm{O}\right.$ nanofluid with different shapes) treatment via a porous semi-annulus using an advanced numerical method. Raza et al. [34] deliberated combined effects of thermal radiation and magnetic field of nanofluid molybdenum disulfide in a wall-changing tube, considering water as a base fluid and MoS2 as nanoparticles of various shapes. Ramalingeswara Rao et al. [35] were concerned with studying radiation absorption and heat source on an unstable MHD Casson fluid past an exponential plate.

The above literature is the motivation behind the main emphasis in studying hydromagnetic boundary layer nanofluid flow past a moving vertical plate consisting of three different nanoparticles $\mathrm{Cu}, \mathrm{Al}_{2} \mathrm{O}_{3}$, and $\mathrm{TiO}_{2}$ containing water as a base fluid in the presence of a uniform moving magnetic field, when the magnetic field fixed to the plate or the fluid. We obtained a closed-form solution using Laplace transform procedure without any limitation. Fluid properties have been discussed for a moving as well as the static plate through graphs for different water-based nanoparticles and also non-dimensional parameters which are involved in the physical problem. 


\section{Materials and Methods}

\subsection{Mathematical formulation.}

Consider an unsteady, free convective, hydromagnetic flow of impulsive water-based nanofluid consisting of nanoparticles $\mathrm{Cu}, \mathrm{Al}_{2} \mathrm{O}_{3}$, and $\mathrm{TiO}_{2}$ through a moving vertical plate under the influence of an unvarying moving magnetic field. The $x$-axis is measured upwards along with the vertical plate, and $y$-axis is exactly normal (perpendicular) to it, as shown in the problem's geometry. A static, moving magnetic field of strength $B_{0}$ is imposed relative to the fluid as well as to the plate. Also, radiative heat flux $q_{r}$ is considered perpendicular to the plate and assumed that the pressure gradient is neglected. The nanoparticles of the nanofluid and the suspended nanoparticles are uniform in size, shape in thermal equilibrium. In order to develop a mathematical model for a closed-form solution, we assume the following assumptions. (i) Initially at $t=0$, the plate is static with a temperature $T_{\infty}$ and surrounding fluid at all the points have the same temperature in the region $y \geq 0$; (ii) At $t \geq 0$, the temperature of the plate increases or decreases $T_{\mathrm{w}}$ once the plate starts moving with the velocity $\mathrm{u}=\lambda \mathrm{u}_{0}$ in the direction of the vertical plate; (iii) The induced magnetic field is negligible; (iv) The density variations in the body force with respect to temperature only; (v) The fluid is flowing only in $x$-axis. The geometry and its physical interpretation are shown in Figure 1.

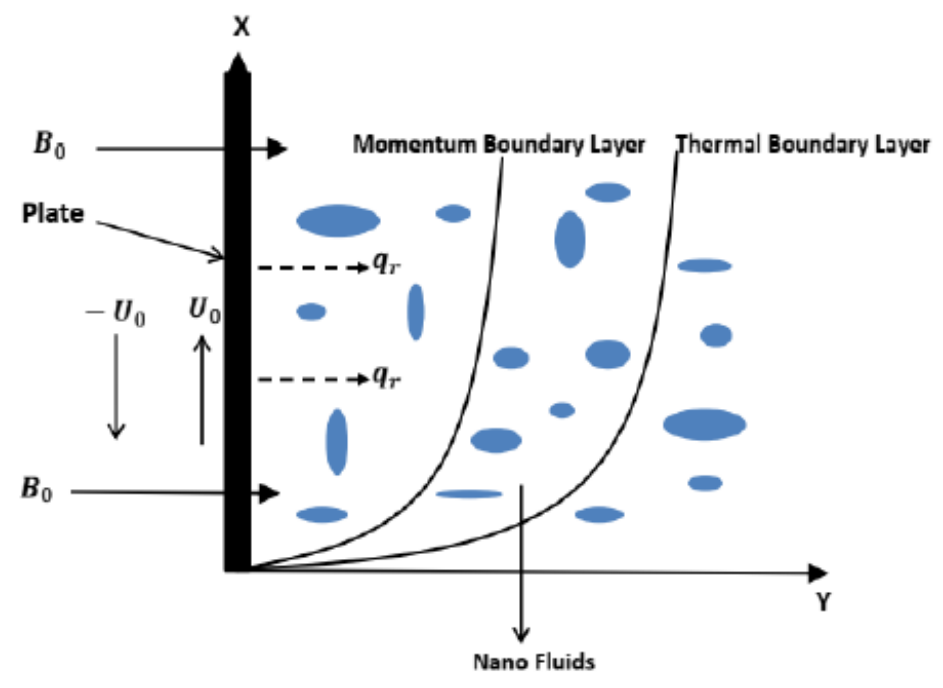

Figure 1. Geometry and coordinate system.

With respect to the above assumptions, the governed PDE's equations are:

$$
\begin{aligned}
& \rho_{n f} \frac{\partial u}{\partial t}=\mu_{n f} \frac{\partial^{2} u}{\partial y^{2}}+g(\rho \beta)_{n f}\left(T-T_{\infty}\right)-\sigma_{n f} B_{0}^{2} u, \\
& \left(\rho c_{p}\right)_{n f} \frac{\partial T}{\partial t}=k_{n f} \frac{\partial^{2} T}{\partial t^{2}}-\frac{\partial q_{r}}{\partial y} .
\end{aligned}
$$

When, $B_{0}$ fixed to an exponentially accelerated plate, Eq.(1) becomes,

$\rho_{n f} \frac{\partial u}{\partial t}=\mu_{n f} \frac{\partial^{2} u}{\partial y^{2}}+g(\rho \beta)_{n f}\left(T-T_{\infty}\right)-\sigma_{n f} B_{0}^{2}\left[u-u_{0} \exp \left(a_{0}^{\prime} t^{\prime}\right)\right]$,

now substitute Eq. (3) in (1), we get 
$\rho_{n f} \frac{\partial u}{\partial t}=\mu_{n f} \frac{\partial^{2} u}{\partial y^{2}}+g(\rho \beta)_{n f}\left(T-T_{\infty}\right)-\sigma_{n f} B_{0}^{2}\left[u-K u_{0} \exp \left(a_{0}^{\prime} t^{\prime}\right)\right]$,

and the corresponding conditions are

$$
\begin{aligned}
t \leq 0: u=0, & T=T_{\infty}, \quad \text { for } y \geq 0, \\
t>0: u=\lambda u_{0}, & T=T_{w}, \quad \text { at } y=0, \\
u \rightarrow 0, & T \rightarrow T_{\infty}, \quad \text { as } y \rightarrow \infty .
\end{aligned}
$$

Here, $K=0,1$ represents when $B_{0}$ fixed to the fluid or to the plate respectively, the plate is stationary when $\lambda=0$ and is in forth and back movement when $\lambda= \pm 1$.

The thermal radiation by Rosseland [1931] approximation is

$$
q_{r}=-\frac{4 \sigma^{*}}{3 k^{*}} \frac{\partial T^{4}}{\partial y} \text {. }
$$

substituting Eqs. (6) in Eq. (2) we get

$$
\frac{\partial T}{\partial t}=\frac{1}{\left(\rho c_{p}\right)_{n f}}\left(k_{n f}+\frac{16 \sigma^{*} T_{\infty}^{3}}{3 k^{*}}\right) \frac{\partial^{2} T}{\partial y^{2}} \text {. }
$$

The thermophysical properties of the nanofluid are given by

$$
\begin{aligned}
& \rho_{n f}=(1-\phi) \rho_{f}+\phi \rho_{s} ;\left(\rho c_{p}\right)_{n f}=(1-\varphi)\left(\rho c_{p}\right)_{f}+\phi\left(\rho c_{p}\right)_{s} ;(\rho \beta)_{n f}=(1-\phi)(\rho \beta)_{f}+\phi(\rho \beta)_{s} ; \\
& \mu_{n f}=\frac{\mu_{f}}{(1-\phi)^{2.5}} ; \sigma_{n f}=\sigma_{f}\left[1+\frac{3(\sigma-1) \phi}{(\sigma+2)-(\sigma-1) \phi}\right] ; k_{n f}=k_{f}\left[\frac{k_{s}+2 k_{f}-2 \phi\left(k_{f}-k_{s}\right)}{k_{s}+2 k_{f}+\phi\left(k_{f}-k_{s}\right)}\right] .
\end{aligned}
$$

Introducing non-dimensional quantities

$$
\eta=\frac{u_{0} y}{v_{f}}, \tau=\frac{u_{0}^{2} t}{v_{f}}, u_{1}=\frac{u}{u_{0}}, \theta=\frac{T-T_{\infty}}{T_{w}-T_{\infty}}, t=\frac{t^{\prime} u_{0}^{2}}{v}, a_{0}=\frac{a^{\prime} v}{u_{0}^{2}} \text {. }
$$

Rewrite Eqs. (4), (5) and (7) using Eqs. (8) and (9), we get in dimensionless form as follows.

$$
\begin{aligned}
& \frac{\partial u_{1}}{\partial \tau}=a_{1} \frac{\partial^{2} u_{1}}{\partial \eta^{2}}+G r a_{2} \theta-M^{2} a_{3}\left(u_{0}-K e^{a_{0} t}\right), \\
& \frac{\partial \theta}{\partial \tau}=a_{4} \frac{\partial^{2} \theta}{\partial \eta^{2}}, \\
& t \leq 0: u_{1}=0, \quad \theta=0, \quad \text { for all } \eta \geq 0, \\
& t>0: u_{1}=\lambda, \quad \theta=1, \quad \text { at } \eta=0, \\
& \quad u_{1} \rightarrow 0, \quad \theta \rightarrow 0, \quad \text { as } \eta \rightarrow \infty
\end{aligned}
$$

where, $a_{1}=\frac{1}{(1-\phi)^{2.5} x_{1}} ; a_{2}=\frac{x_{2}}{x_{1}} ; a_{3}=\frac{x_{5}}{x_{1}} ; a_{4}=\frac{1}{x_{3} \operatorname{Pr}}\left(x_{4}+N r\right) ; x_{1}=\left[(1-\phi)+\phi\left(\frac{\rho_{s}}{\rho_{f}}\right)\right]$; $x_{2}=\left[(1-\phi)+\phi \frac{(\rho \beta)_{s}}{(\rho \beta)_{f}}\right] ; x_{3}=\left[(1-\phi)+\phi \frac{\left(\rho c_{p}\right)_{s}}{\left(\rho c_{p}\right)_{f}}\right] ; x_{4}=\left[\frac{k_{s}+2 k_{f}-2 \phi\left(k_{f}-k_{s}\right)}{k_{s}+2 k_{f}+\phi\left(k_{f}-k_{s}\right)}\right]$; $x_{5}=\left[1+\frac{3\left(\frac{\sigma_{s}}{\sigma_{f}}-1\right) \phi}{\left(\frac{\sigma_{s}}{\sigma_{f}}+2\right)-\left(\frac{\sigma_{s}}{\sigma_{f}}-1\right) \phi}\right] ; M^{2}=\frac{\sigma_{f} B_{0}^{2} v_{f}}{\rho_{f} u_{0}^{2}}$ is the magnetic parameter; $N r=\frac{16 \sigma^{*} T_{\infty}^{3}}{3 k_{f} k^{*}}$ is the 
radiation parameter; $\operatorname{Pr}=\frac{\mu_{f} c_{p}}{k_{f}}$ is the Prandtl number, and $G r=\frac{g \beta_{f} v_{f}\left(T_{w}-T_{\infty}\right)}{u_{0}^{3}}$ is the Grashof number.

The dimensionless equations and conditions (10)-(12) are solved using the Laplace transform technique

$$
\begin{aligned}
& u_{1}(\eta, \tau)=\frac{\left(\lambda-b_{2}\right)}{2}\left[\exp (y \sqrt{\gamma \tau}) \operatorname{erfc}\left(\frac{\eta}{2 \sqrt{\tau}}+\sqrt{\gamma \tau}\right)+\exp (-y \sqrt{\gamma \tau}) \operatorname{erfc}\left(\frac{\eta}{2 \sqrt{\tau}}-\sqrt{\gamma \tau}\right)\right] \\
& +\frac{b_{2} e^{b \tau}}{2}\left[\exp (y \sqrt{\gamma+b}) \operatorname{erfc}\left(\frac{\eta}{2 \sqrt{\tau}}+\sqrt{(\gamma+b) \tau}\right)+\exp (-y \sqrt{\gamma+b}) \operatorname{erfc}\left(\frac{\eta}{2 \sqrt{\tau}}-\sqrt{(\gamma+b) \tau}\right)\right] \\
& -\frac{b_{3} e^{a_{0} \tau}}{2}\left[\exp \left(y \sqrt{\gamma+a_{0}}\right) \operatorname{erfc}\left(\frac{\eta}{2 \sqrt{\tau}}+\sqrt{\left(\gamma+a_{0}\right) \tau}\right)+\exp \left(-y \sqrt{\gamma+a_{0}}\right) \operatorname{erfc}\left(\frac{\eta}{2 \sqrt{\tau}}-\sqrt{\left(\gamma+a_{0}\right) \tau}\right)\right] \\
& +b_{3} e \exp (-\gamma \tau) \operatorname{erfc}\left(\frac{\eta}{2 \sqrt{\tau}}\right)+b_{2} \operatorname{erfc}\left(\frac{\eta \sqrt{\alpha}}{2 \sqrt{\tau}}\right)+b_{3} \exp \left(a_{0} \tau\right)-b_{3} \exp (-\gamma \tau) \\
& \frac{-b_{2} e^{b \tau}}{2}\left[\exp (\eta \sqrt{\alpha b}) \operatorname{erfc}\left(\frac{\eta \sqrt{\alpha}}{2 \sqrt{\tau}}+\sqrt{b \tau}\right)+\exp (-\eta \sqrt{\alpha b}) \operatorname{erfc}\left(\frac{\eta \sqrt{\alpha}}{2 \sqrt{\tau}}-\sqrt{b \tau}\right)\right] \\
& \theta(\eta, \tau)=\operatorname{erfc}\left(\frac{\eta \sqrt{\alpha}}{2 \sqrt{\tau}}\right) .
\end{aligned}
$$

Where, $\alpha=\frac{1}{a_{4}}, \gamma=a_{3} M^{2}, a_{5}=\frac{a_{2} a_{4}}{a_{1}-a_{4}}, b=\frac{a_{3} a_{4} M^{2}}{a_{1}-a_{4}}, b_{1}=M^{2} a_{3} K, b_{2}=\frac{G r a_{5}}{b}, b_{3}=\frac{b_{1}}{a_{0}+\gamma}$.

The skin-friction and the Nusselt number in the non-dimensional form are

$$
\begin{aligned}
& \tau=-\left[\frac{d u_{1}}{d \eta}\right]_{\eta=0}=\left(\lambda-b_{2}\right)\left(\sqrt{\gamma} \operatorname{erf}(\sqrt{\gamma \tau})+\frac{\mathrm{e}^{-\gamma \tau}}{\sqrt{\pi \tau}}\right)+b_{2} \exp (b \tau)(\sqrt{\gamma+b} \operatorname{erf}(\sqrt{(\gamma+b) \tau})) \\
& -b_{3} \exp \left(a_{0} \tau\right)\left(\sqrt{\gamma+a_{0}} \operatorname{erf}\left(\sqrt{\left(\gamma+a_{0}\right) \tau}\right)\right)-b_{3} \frac{e^{-\gamma \tau}}{\sqrt{\pi \tau}}-b_{2} \exp (b \tau)(\sqrt{\alpha b} \operatorname{erf}(\sqrt{b \tau}))-b_{2} \sqrt{\frac{\alpha}{\pi \tau}} . \\
& N u=-\left[\frac{d \theta}{d \eta}\right]_{\eta=0}=-\sqrt{\frac{\alpha}{\pi \tau}} .
\end{aligned}
$$

\section{Results and Discussion}

To understand the physics of the model, an analytical study has been conducted, and results are presented in the form of plots. The fluid properties such as velocity, skin friction, and heat transfer rate are discussed for different water-based nanoparticles and most significant non-dimensional parameters like magnetic parameter $\left(M^{2}\right)$, radiation parameter $(N r)$, volume fraction parameter $(\phi)$, and thermal Grashof number $(G r)$ in figures 2-19. Here, the values of volume fraction are taken from 0 to 0.2 . To verify the obtained results are correct or not, a comparative study has been done when $B_{0}$ fixed to the fluid $(K=0)$ with the available literature in the absence of a few non-dimensional parameters and found good agreement. This analysis $\lambda=-1$ represents the vertical plate's downward motion, $\lambda=1$ represents the upward motion 
of the vertical plate, and $\lambda=0$ represents the static plate. The thermophysical features of waterbased nanoparticles are given in Table.1.

The transverse velocity profile behavior subjected to different nanofluids (Water-based nanoparticles $\mathrm{Cu}, \mathrm{Al}_{2} \mathrm{O}_{3}$, and $\mathrm{TiO}_{2}$ ) with the same volume fraction $K=0$ and $K=1$ is shown in figures 2 and 3. The velocity profile decreases for Copper, Aluminum Oxide, and Titanium dioxide due to an enhancement in dynamic viscosity from these figures. Also seen that copper has the highest variation and Titanium dioxide having the least variation in velocity $K=0$ and $K=1$. Figures 4 and 5 illustrates velocity variations for distinct values of the magnetic parameter and understands that as the magnetic field increases, the velocity gets increase for $K=0$ and $K=1$. The reason is, the inclined magnetic field will result in a Lorentz force because it has only one component in the fluid direction, which helps to accelerate the velocity distribution. Also seen that copper has the lowest variation, and titanium dioxide having the highest variation in velocity profiles. The effect of thermal Grashof number on transverse velocity is presented in figures 6 and 7 and observed that the velocity distribution increases when copper has the highest variation and titanium dioxide have the least variation in velocity. The physics behind it is that as increasing Grashof number, the buoyancy force and its allied forces become stronger, suppressing the viscous force. Ultimately, because of this viscous force, fluid gets accelerated because Grashof number is the ratio of thermal buoyancy force to the viscous force.

Figures 8 and 9 show the effect of solid volume fraction on dimensionless velocity and noticed that fluid flow gets accelerated as increasing in both cases for moving and static plate because the viscous forces become weak when momentum boundary layer thickness enhances in a rise in the velocity profile. The effect of the radiation parameter on velocity is drawn for $K=0$ and $K=1$ in figures 10 and 11 and noticed that velocity profiles enhance as the thermal radiation increases in fluid. The physics behind it is, the bonds between fluid components become weak due to the enhancement of higher energy transport. Thus velocity gets accelerated for a moving plate as well as a static plate.

Figures 12-19 show the variation in Skin friction, heat transfer rate for different nanoparticles as well the magnetic parameter, radiation parameter, fraction parameter $(\phi)$, and thermal Grashof number. Figure 12 shows that the local skin friction coefficient increases for different nanoparticles (Water-based Copper, Aluminum oxide, and Titanium dioxide) when $K=0$ moving like a well static plate. An opposite behavior is seen when $K=1$ for moving as the well static plate is observed from Fig.13. Similarly, from figure 14, as the magnetic parameter increases, the local skin friction coefficient decreases when $K=0$ for the moving plate and static plate, and from Fig.15, the skin friction coefficient increases when $K=1$ for the moving plate and static plate. The effect of Grashof number for $K=0$ and $K=1$ on the local skin friction coefficient is illustrated in figures 17 and 18 for the moving and static plate. It is revealed that the skin friction coefficient decreases as $G r$ it increases for $\lambda= \pm 1$ and $\lambda=0$. Figure 18 illustrate that, as increasing $N r$ values increases heat transfer rate when $K=0$ for moving plate as well as the static plate. The physics behind it is, the temperature gradient becomes more powerful dominant when thermal radiation has larger values. This domination increases the rate of heat transfer. The effect of Pr on the rate of heat transfer is presented when $K=1$ for the moving plate as well as a static plate in figure 19 , and it is noticed that there is a heat transfer for higher Pr values due to thermal conductivity relatively small for higher Prandtl values. Hence conduction of heat becomes weak for such fluids. 
Table 1. Thermophysical properties of water and nanoparticles.

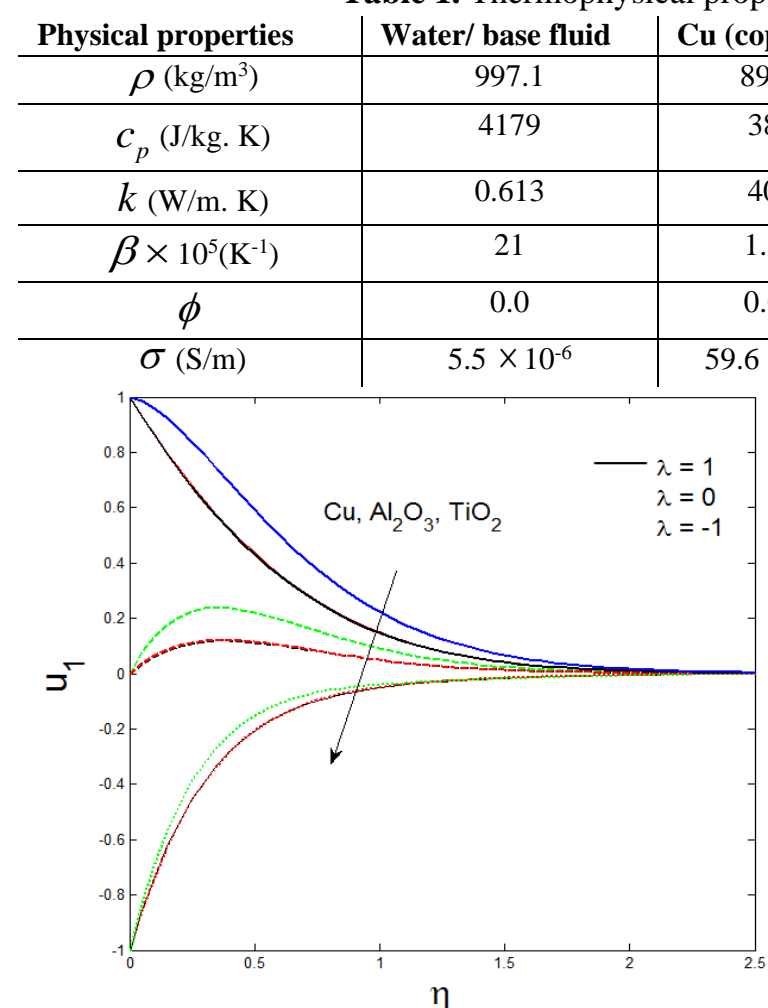

Figure 2. Velocity variation of various nanofluids,

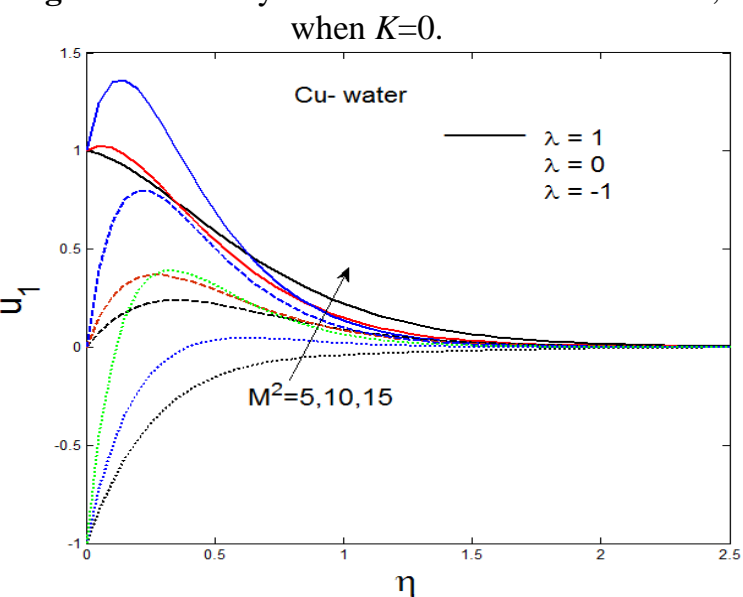

Figure 4. Velocity variation of $M^{2}$, when $K=0$.

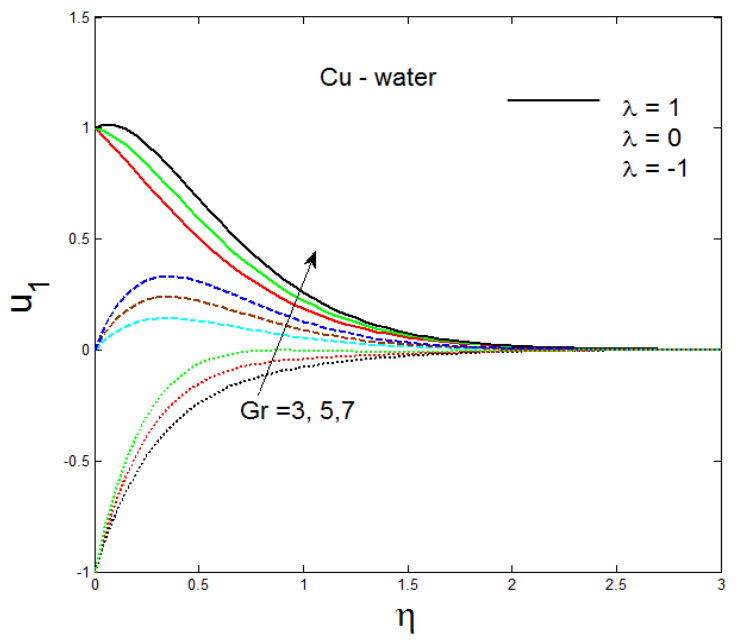

Figure 6. Velocity variation of $G r$, when $K=0$.
$\mathrm{TiO}_{2}$ (titanium oxide)

\begin{tabular}{|c|c}
\hline $\mathbf{A l}_{2} \mathbf{O}_{3}$ (alumina) & $\mathbf{T i O}_{2}$ (titanium oxide) \\
\hline 3970 & 4250 \\
\hline 765 & 686.2 \\
\hline 40 & 8.9538 \\
\hline 0.85 & 0.90 \\
\hline $35 \times 10^{6}$ & 0.2 \\
\hline
\end{tabular}

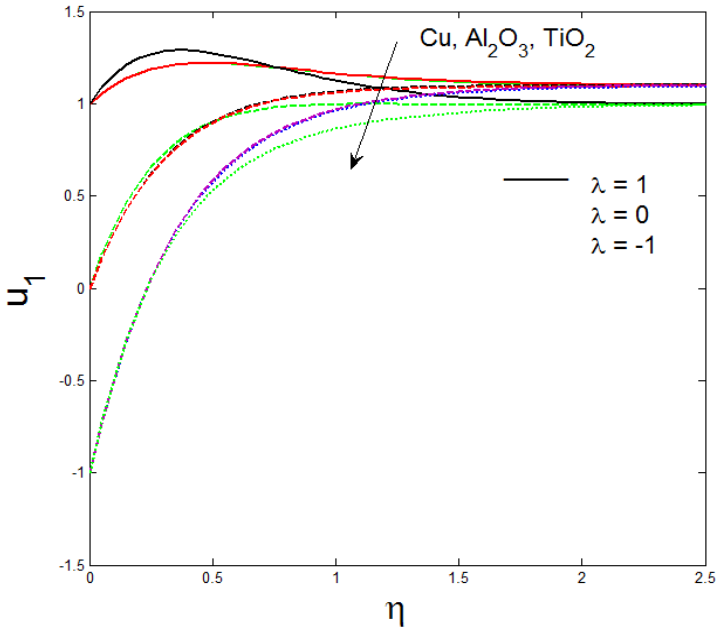

Figure 3. Velocity variation of various nanofluids,

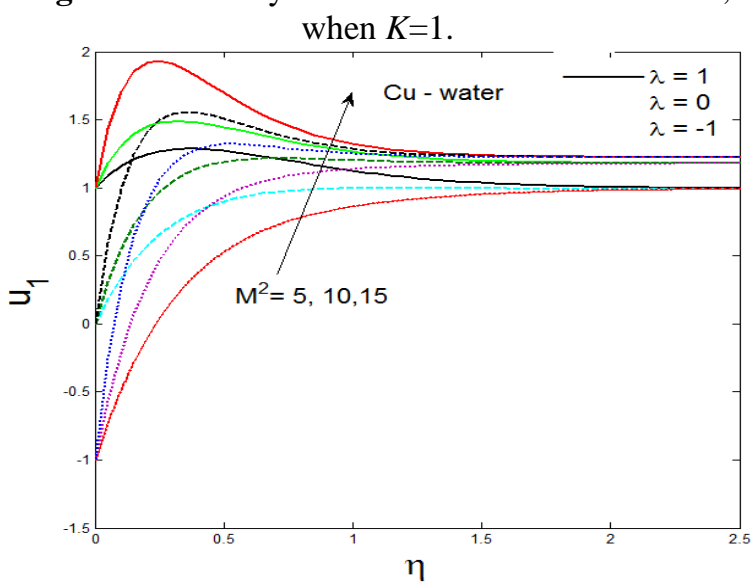

Figure 5. Velocity variation of $M^{2}$, when $K=1$.

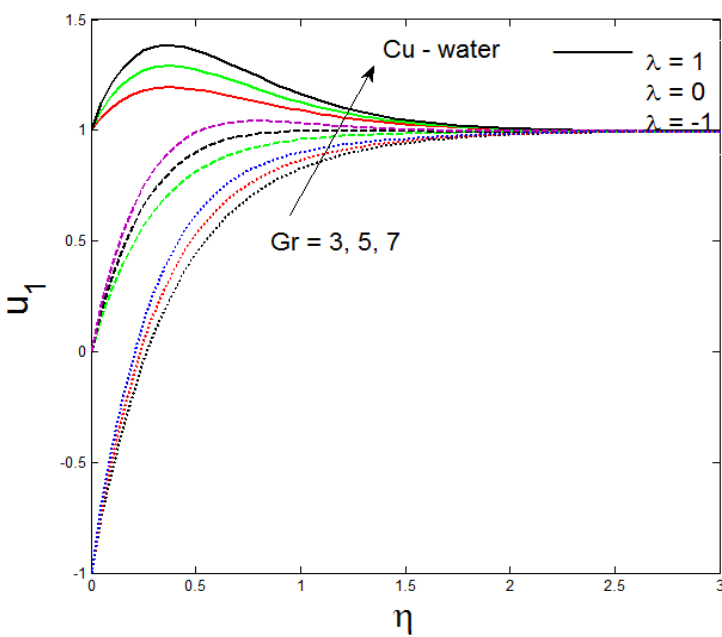

Figure 7. Velocity variation of $G r$, when $K=1$. 


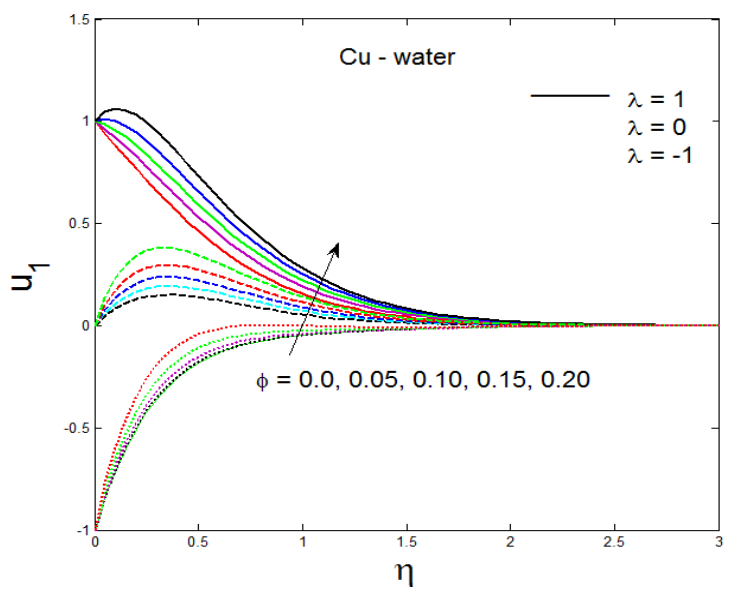

Figure 8. Velocity profile for different $\phi$ when

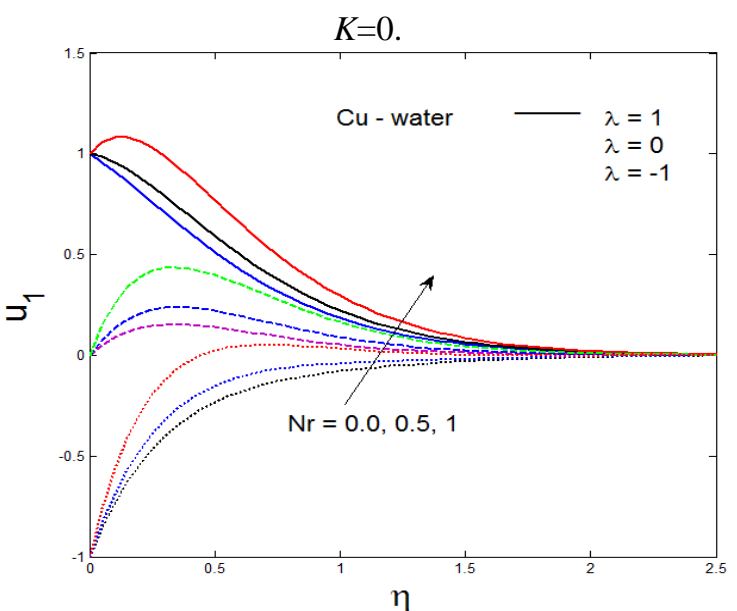

Figure 10. Velocity variation of $N r$, when $K=0$.

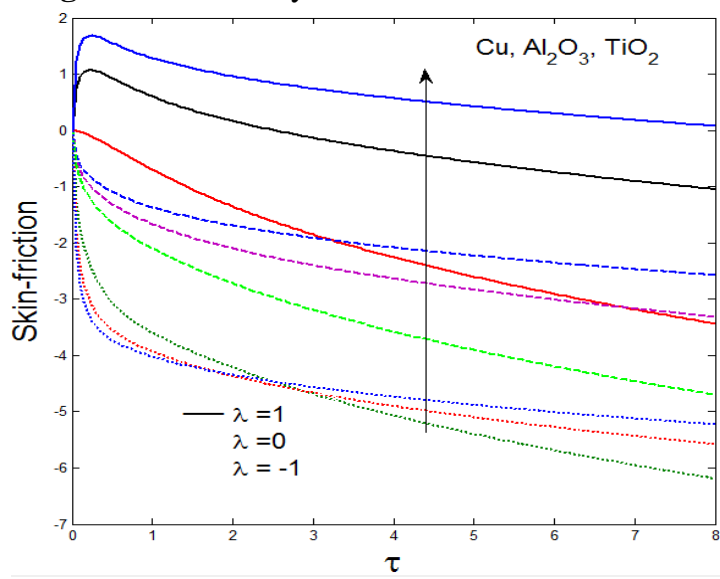

Figure 12. Skin-friction of various nanoparticles when $K=0$.

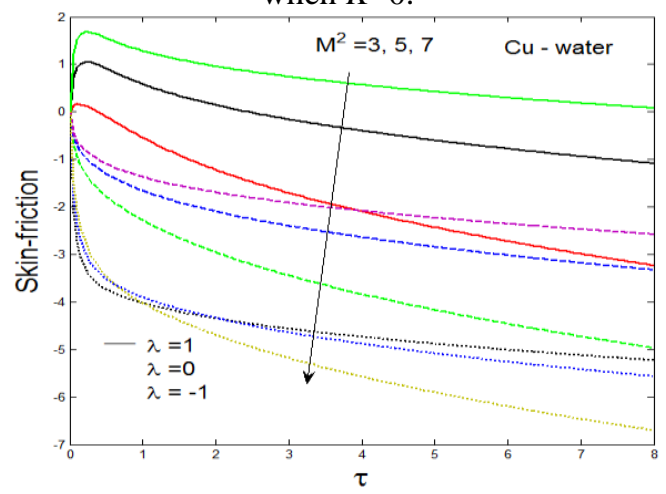

Figure 14. Skin-friction for various $M^{2}$, when $K=0$.

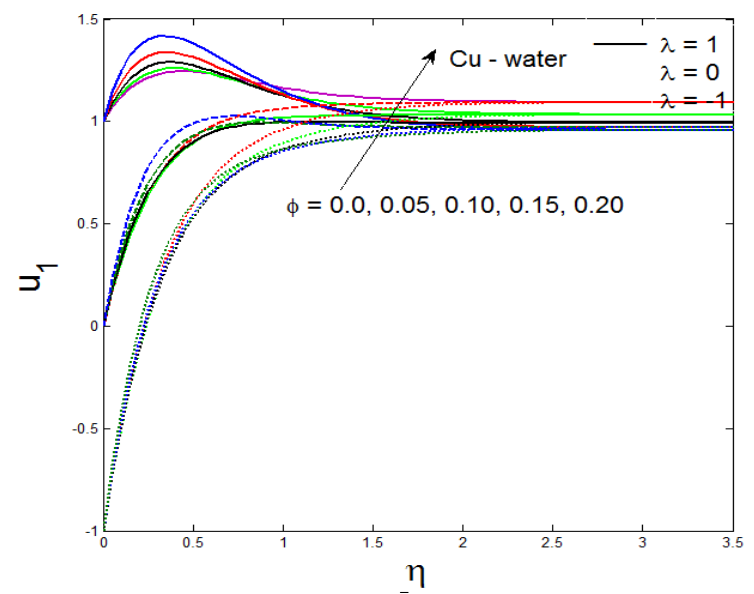

Figure 9. Velocity profile for different $\phi$ when

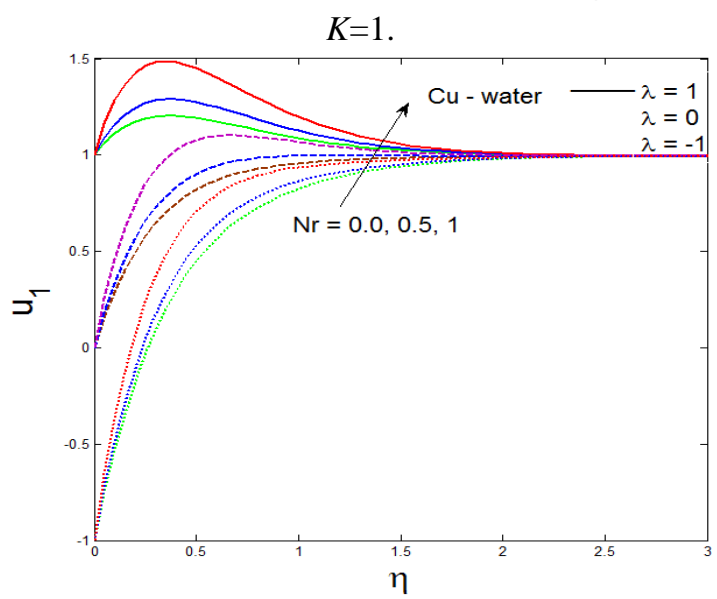

Figure 11. Velocity variation of $N r$, when $K=1$.

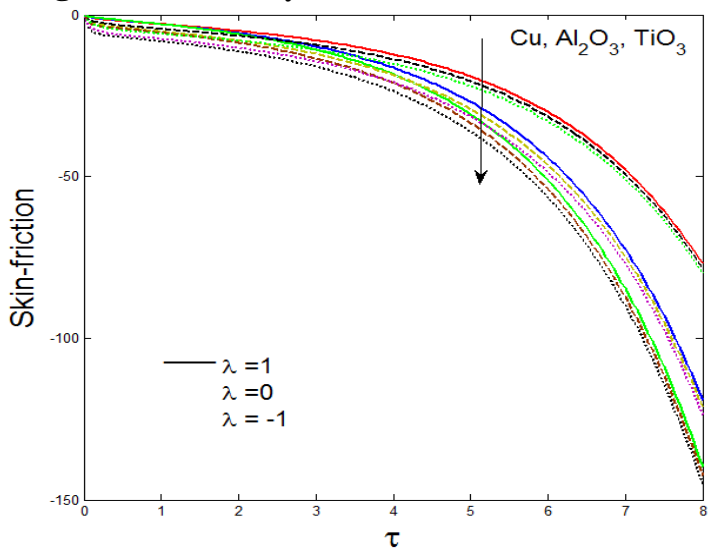

Figure 13. Skin-friction of various nanoparticles when $K=1$.

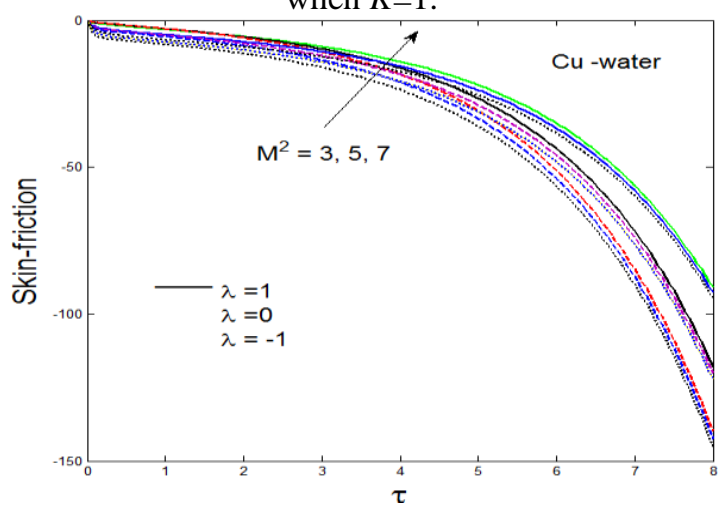

Figure 15. Skin-friction for various $M^{2}$, when $K=1$. 


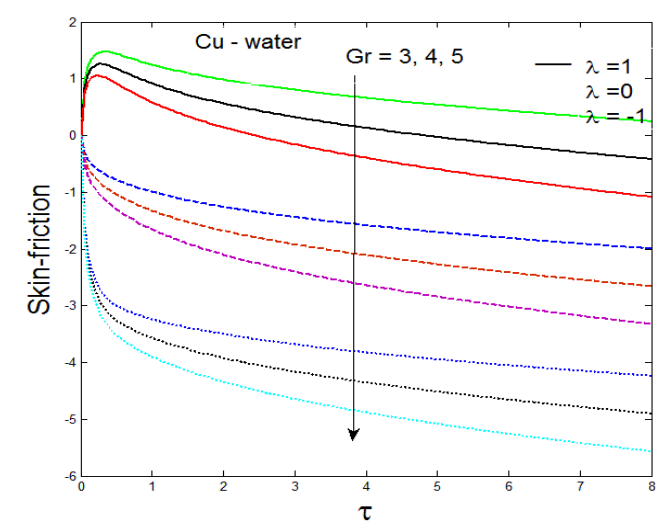

Figure 16. Skin-friction for various $G r$, when $K=0$.

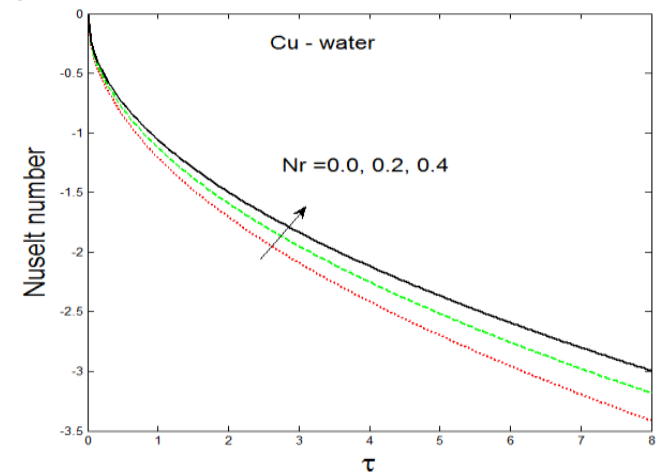

Figure 18. Nusselt number for various $N r$, when $K=0$.

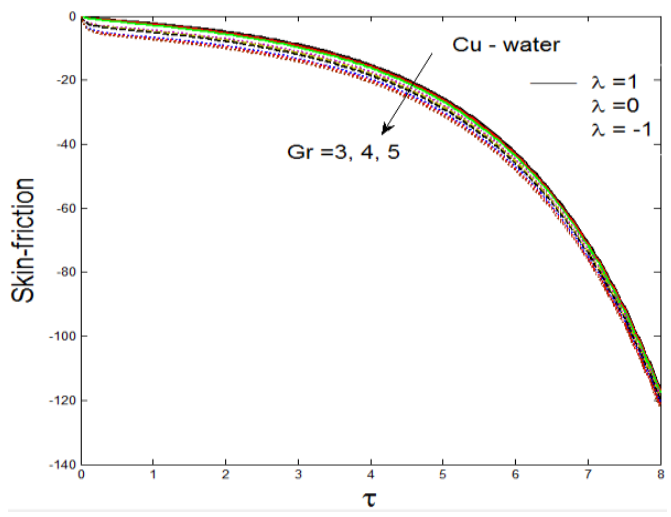

Figure 17. Skin-friction for various $G r$, when $K=1$.

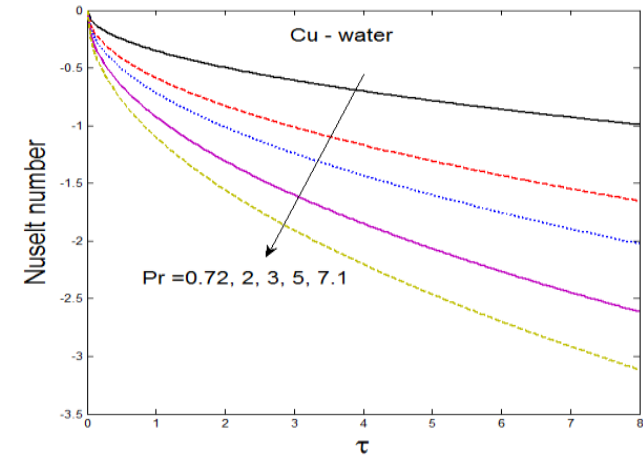

Figure 19. Nusselt number for various $P r$, when $K=1$.

\section{Conclusions}

A parametric study has been done to obtain an exact solution for an unsteady, free convective, hydromagnetic flow of impulsive water-based nanofluid consisting of $\mathrm{Cu}, \mathrm{Al}_{2} \mathrm{O}_{3}$, and $\mathrm{TiO}_{2}$ past a moving vertical plate in the occurrence of a uniform transverse magnetic field and radiative heat flux when $K=0$ and $K=1$ for moving as well as the static plate. The governing PDE's along its initial and BC's are solved using Laplace transform technique after converting into dimensionless form. The non-dimensional parameters associated with momentum and energy equations are illustrated with the help of plots for velocity, Skin friction, and Nusselt number. Based on this analysis, the following conclusions are drawn: (i) The velocity accelerated for a moving plate as well as a static plate for all nanofluids (water-based nanofluid consisting of $\mathrm{Cu}, \mathrm{Al}_{2} \mathrm{O}_{3}$, and $\mathrm{TiO}_{2}$ ), as well as the magnetic parameter, Grashof number, Radiation parameter, Volume fraction parameter enhances when $K=0$ and $K=1$. A reverse trend is observed in nanofluids when $K=0$ and $K=1$; (ii) The skin friction coefficient increases for a moving plate as well as a static plate when $K=0$ and decreases when $K=1$ for all nanofluids; (iii) The skin friction coefficient decreases for moving and static plate, when $K=0$ and increases, when $K=1$ as the magnetic parameter increases; (iv) The skin friction coefficient decreases for a moving plate and static plate when $K=0$ and increases when $K=1$ as thermal Grashof number increases; (v) The rate of heat transfer enhances for a moving plate as well as a static plate when $K=0$ as $N r$ increases, and it decreases when $K=1$ as Prandtl number increases.

\section{Funding}

This research received no external funding. 


\section{Acknowledgments}

The authors would like to thank the anonymous reviewers for their valuable comments and suggestions to improve the paper's quality.

\section{Conflicts of Interest}

The authors declare no conflict of interest.

\section{References}

1. Choi, S.U.S. Enhancing thermal conductivity of fluids with nanoparticles developments and applications of non-Newtonian flows. ASME FED 231/MD 1995, 66, 99-105.

2. Eastman, J.A.; Choi, S.U.S.; Li, S.; Yu, W.; Thompson, L.J. Anomalously increased effective thermal conductivities of ethylene glycol-based nanofluids containing copper nanoparticles. Applied Physics Letters 2001, 78, 718-720, https://doi.org/10.1063/1.1341218.

3. Das, S.K.; Putra, N.; Thiesen, P.; Roetzel, W. Temperature dependence of thermal conductivity enhancement for nanofluids. Journal of Heat Transfer 2003, 125, 567-574, https://doi.org/10.1115/1.1571080.

4. Das, S.K.; Choi, S.U. A review of Heat Transfer in nanofluids. Advances in Heat Transfer 2009, 46, 81-197, https://doi.org/10.1016/S0065-2717(08)41002-X.

5. Kuznetsov, A.V.; Nield, D.A. Natural convective boundary layer flow of a nanofluid past a vertical plate. International Journal of Thermal Sciences, 2010, 49, 243-247. https://doi.org/10.1016/j.ijthermalsci.2013.10.007.

6. Srinivas, S.; Gupta, A.; Kandoi, A. Modelling and simulation of au-water nanofluid flow in wavy channels. Frontiers in Heat and Mass Transfer 2015, 5.

7. Chamkha, A.J.; Aly, A.M. MHD free convection flow of a nanofluid past a vertical plate in the presence of heat generation/absorption effects. Chemical Engineering Communications 2010, 198, 425-441. https://doi.org/10.1080/00986445.2010.520232.

8. Jahan, S.; Sakidin, H.; Nazar, R and Pop, I. Analysis of heat transfer in nanofluid past a convectively heated permeable stretching/shrinking sheet with regression and stability analyses. Results in Physics 2018, 10, 395-405, https://doi.org/10.1016/j.rinp.2018.06.021.

9. Hamad, M.A.A.; Pop, I. Unsteady MHD free convection flow past a vertical permeable flat plate in a rotating frame of reference with constant heat source in a nanofluid. Heat and Mass Transfer 2011, 47, 11517-1524, https://doi.org/10.1007/s00231-011-0816-6.

10. Sheikholeslami, M.; Hatami, M.; Domairry, G. Numerical simulation of two phase unsteady nanofluid flow and heat transfer between parallel plates in presence of time dependent magnetic field. Journal of the Taiwan Institute of Chemical Engineers 2015, 46, 43-50, https://doi.org/10.1016/j.jtice.2014.09.025.

11. Shankar Goud, B.; Pramod Kumar, P.; Malga, B.S. Effect of Heat source on an unsteady MHD free convection flow of Casson fluid past a vertical oscillating plate in porous medium using finite element analysis. Partial Differential Equations in Applied Mathematics 2020, 2. https://doi.org/10.1016/j.padiff.2020.100015.

12. Islam, M.R.; Nasrin, S.; Alam, M.M. Unsteady electromagnetic free convection micropolar fluid flow through a porous medium along a vertical porous plate, Journal of Applied Sciences 2020, 10, 701-718. https://doi.org/10.4236/ojapps.2020.1011049.

13. Sheikholeslami, M.; Bandpya, M.G.; Ganji, D.D. Numerical investigation of MHD effects on $\mathrm{Al}_{2} \mathrm{O}_{3} \mathrm{water}$ nanofluid flow and heat transfer in a semi-annulus enclosure using LBM. Energy 2013, 60 501-510. https://doi.org/10.1016/j.energy.2013.07.070.

14. Sheikholeslami, M.; Gorji Bandpy, M.; Ellahi, R.; Zeeshan, A. Simulation of MHD CuO-water nanofluid flow and convective heat transfer considering Lorentz forces. Journal of Magnetism and Magnetic Materials, 2014, 369, 69-80. https://doi.org/10.1016/j.jmmm.2014.06.017.

15. Molli, S.; Naikoti, K. MHD natural convective flow of $\mathrm{Cu}-$ Water nanofluid over a past infinite vertical plate with the presence of time dependent boundary condition. International Journal of Thermofluid Science and Technology 2020, 7, https://doi.org/10.36963/ijtst.2020070404.

16. Animasaun, I.L.; Koriko, O.K.; Adegbie, K.S.; Babatunde, H.A.; Ibraheem, R.O.; Sandeep, N.; Mahanthesh, B. Comparative analysis between $36 \mathrm{~nm}$ and $47 \mathrm{~nm}$ alumina-water nanofluid flows in the presence of Hall effect. Journal of Thermal Analysis and Calorimetry 2019, 135, 873-886. https://doi.org/10.1007/s10973-018-7379-4.

17. Hussanan, A.; Khan, I.; Hashim, H.; Anuar, M.K.; Ishak, N.; Sarif, N.M.; Salleh, M.Z. Unsteady MHD flow of some nanofluids past an accelerated vertical plate embedded in a porous medium. Jurnal Teknologi 2016, 78, https://doi.org/10.11113/jt.v78.4900. 
18. Abbas, W.; Magdy, M.M. Heat and Mass Transfer Analysis of Nanofluid Flow Based on Cu, Al2O3, and TiO2 over a Moving Rotating Plate and Impact of Various Nanoparticle Shapes. Mathematical Problems in Engineering, 2020, 2020, 1-12, https://doi.org/10.1155/2020/9606382.

19. Hayat, T.; Ahmed, B.; Abbasi, F.M.; Alsaedi, A. Hydromagnetic peristalsis of water based nanofluids with temperature dependent viscosity: A comparative study. Journal of Molecular Liquids 2017, 234, 324-329, https://doi.org/10.1016/j.molliq.2017.03.080.

20. Zhou, X.; Jiang, Y.; Li, X.; Cheng, K.; Huai, X.; Zhang, X.; Huang, H. Numerical investigation of heat transfer enhancement and entropy generation of natural convection in a cavity containing nano liquid-metal fluid. International Communications in Heat and Mass Transfer 2019, 106, 46-54. https://doi.org/10.1016/j.icheatmasstransfer.2019.05.003.

21. Anwar, T.; Kumam, P.; Khan, I.; Watthayu, W. Heat Transfer Enhancement in Unsteady MHD Natural Convective Flow of CNTs Oldroyd-B Nanofluid under Ramped Wall Velocity and Ramped Wall Temperature. Entropy 2020, 22, https://doi.org/10.3390/e22040401.

22. Sandeep, N.; Sugunamma, V. Radiation and inclined magnetic field effects on unsteady hydromagnetic free convection flow past an impulsively moving vertical plate in a porous medium. Journal of Applied Fluid Mechanics 2014, 7, 275-286, https://doi.org/10.36884/jafm.7.02.19431.

23. Nandkeolyar, R.; Das, M. MHD free convective radiative flow past a flat plate with ramped temperature in the presence of an inclined magnetic field. Computational and Applied Mathematics 2015, 34, 109-123, https://doi.org/10.1007/s40314-013-0107-6.

24. Rushi Kumar, B.; Sravan Kumar, T.; Vijaya Kumar, A.G. Thermal diffusion and radiation effects on unsteady free convection flow in the presence of magnetic field fixed relative to the fluid or to the plate. Frontiers in Heat and Mass Transfer 2015, 6, 6-12, https://doi.org/10.5098/hmt.6.12.

25. Mishra, P.; Tripathi, S. Effect of non-uniform heat source and radiation on unsteady MHD free convection flow past an infinite heated vertical plate in porous medium. E-Jurnal Matematika 2020, 9, 219-228, https://doi.org/10.24843/mtk.2020.v09.i04.p302.

26. Chiranjeevi, B.; Valsamy, P.; Vidyasagar, G. Radiation absorption on MHD free convective laminar flow over a moving vertical porous plate, viscous dissipation and chemical reaction with suction under the influence of transverses magnetic field. Materials Today: Proceedings 2020. https://doi.org/10.1016/j.matpr.2020.01.488.

27. Suneetha, K.; Ibrahim, S.; Reddy, G.; Kumar, P. Variable temperature and concentration impacts on radiative chemically magneto hydrodynamic viscoelastic fluid flow through porous moving plate. Mathematical Modelling of Engineering Problems 2020, 7, 187-195, https://doi.org/10.18280/mmep.070203.

28. Turkyilmazoglu, M.; Pop, I. Heat and mass transfer of unsteady natural convection flow of some nanofluids past a vertical infinite flat plate with radiation effect. International Journal of Heat and Mass Transfer 2013, 59, 167-171, https://doi.org/10.1016/j.ijheatmasstransfer.2012.12.009.

29. Sheikholeslami, M.; Ganji, D.D.; Javedb, M.Y.; Ellahi, R. Effect of thermal radiation on Magnetohydrodynamics nanofluid flow and heat transfer by means of Two Phase model. Journal of Magnetism and Magnetic Materials 2015, 374, 36-43, https://doi.org/10.1016/j.jmmm.2014.08.021.

30. Satya Narayana, P.; Venkateswarlu, B.; Venkataramana, S. Thermal radiation and heat source effects on a MHD nanofluid past a vertical plate in a rotating system with porous medium. Heat Transfer Resources 2015, 44, 1-19.

31. Pandit, K.K.; Sarma, D.; Singh, S.I. A study of chemically reactive species and thermal radiation effects on an unsteady MHD free convection flow through a porous medium past a flat plate with ramped wall temperature. International Journal of Applied Mechanics and Engineering 2017, 22, 945-964. https://doi.org/10.1515/ijame-2017-0061.

32. Wakif, A.; Boulahia, Z.; Ali, F.; Eid, M.R.; Sehaqui, R. Numerical analysis of the unsteady natural convection MHD couette nanofluid flow in the presence of thermal radiation using single and two-phase nanofluid models for $\mathrm{Cu}-$ Water nanofluids. International Journal of Applied and Computational Mathematics 2018, 4, 81-95, https://doi.org/10.1007/s40819-018-0513-y.

33. Sheikholeslami, M.; Shafee, A.; Ramzan, M.; Li, Z. Investigation of Lorentz forces and radiation impacts on nanofluid treatment in a porous semi annulus via Darcy law. Journal of Molecular Liquids 2018, 272, 814. https://doi.org/10.1016/j.molliq.2018.09.016.

34. Raza, J.; Oudina,M.F.; Chamkha, A. Magneto-hydrodynamic flow of molybdenum disulfide nanofluid in a channel with shape effects. Multidiscipline Modeling in Materials and Structures 2019, 15, 737-757. https://doi.org/10.1108/mmms-07-2018-0133.

35. Ramalingeswara Rao, S.; Vidyasagar, G.; Deekshitulu, G.V.S.R. Unsteady MHD free convection Casson fluid flow past an exponentially accelerated infinite vertical porous plate through porous medium in the presence of radiation absorption with heat generation/absorption. Materials Today: Proceedings 2020. https://doi.org/10.1016/j.matpr.2020.07.554. 